\title{
mTOR downstream effectors, 4EBP1 and eIF4E, are overexpressed and associated with HPV status in precancerous lesions and carcinomas of the uterine cervix
}

\author{
ARISTIDIS ASIMOMYTIS $^{1}$, MARIA KARANIKOU ${ }^{1}$, ALEXANDER RODOLAKIS ${ }^{2}$, \\ ANNA VAIOPOULOU ${ }^{1}$, PARASKEVI TSETSA ${ }^{2}$, GEORGE CREATSAS ${ }^{3}$, THEODOROS STEFOS ${ }^{4}$, \\ ARISTIDIS ANTSAKLIS ${ }^{2}$, EFSTRATIOS PATSOURIS ${ }^{1}$ and GEORGE Z. RASSIDAKIS ${ }^{1,5}$
}

${ }^{1}$ First Department of Pathology, National and Kapodistrian University of Athens, School of Medicine, GR-11527 Athens;
${ }^{2}$ First Department of Obstetrics and Gynecology, National and Kapodistrian University of Athens,
Alexandra Maternity Hospital; ${ }^{3}$ Second Department of Obstetrics and Gynecology,
National and Kapodistrian University of Athens, Aretaieion Hospital, GR-11528 Athens;
${ }^{4}$ Department of Obstetrics and Gynecology, University of Ioannina, School of Medicine, GR-45110 Ioannina, Greece;
${ }^{5}$ Department of Pathology and Cytology, Karolinska University Hospital and Karolinska Institute, Solna 17176, Sweden

Received May 20, 2015; Accepted June 7, 2016

DOI: $10.3892 / 01.2016 .5056$

\begin{abstract}
The present study aims to investigate the expression levels of two critical mammalian target of rapamycin (mTOR) downstream effectors, 4E binding protein 1 (4EBP1) and eukaryotic initiation factor 4E (eIF4E) proteins, in precancerous squamous intraepithelial lesions and cancer of the uterine cervix, and their association with human papilloma virus (HPV) infection status. Uterine cervical biopsies from 73 patients were obtained, including 40 fresh-frozen samples and 42 archival formalin-fixed, paraffin-embedded tissue specimens. Whole protein extracts were analyzed for the expression of 4EBP1 and eIF4E proteins using western blotting. In addition, distribution of 4EBP1 and eIF4E protein expression and 4EBP1 phosphorylation (P-4EBP1) were analyzed by immunohistochemistry in archival tissues and correlated with the degree of dysplasia. The presence of high-risk HPV (HR-HPV) types was assessed by polymerase chain reaction. Using western blot analysis, high expression levels of 4EBP1 and eIF4E were observed in all uterine cervical carcinomas, which significantly correlated with the degree of dysplasia. By immunohistochemistry, overexpression of 4EBP1 and eIF4E was detected in 20 of 21 (95\%) and 17 of $21(81 \%)$ samples, respectively, in patients with high-grade dysplasia and carcinomas, compared with 1 of $20(5 \%)$ and 2 of $20(10 \%)$ samples, respectively, in patients with low-grade
\end{abstract}

Correspondence to: Professor George Z. Rassidakis, Department of Pathology and Cytology, Karolinska University Hospital and Karolinska Institute, Solna 17176, Sweden

E-mail: grassidakis@hotmail.com georgios.rassidakis@karolinska.se

Key words: 4EBP1, eIF4E, LG-SIL, HG-SIL, Ca, HPV lesions or normal histology. All 4EBP1-positive cases tested were also positive for P-4EBP1. Furthermore, overexpression of 4EBP1 and eIF4E significantly correlated with the presence of HR-HPV oncogenic types. The present study demonstrated that critical effectors of mTOR signaling, which control protein synthesis initiation, are overexpressed in cervical high-grade dysplasia and cancer, and their levels correlate with oncogenic HPV types. These findings may provide novel targets for investigational therapeutic approaches in patients with cancer of the uterine cervix.

\section{Introduction}

Cervical cancer $(\mathrm{Ca})$ is the second most common type of cancer (after breast cancer) in women worldwide. Globally, the mortality ratio is $52 \%$, with $\sim 275,000$ mortalities from $\mathrm{Ca}$ annually (1). Estimated age-adjusted $\mathrm{Ca}$ mortality rates reach $10 / 100,000$ women in developing countries, as opposed to $3 / 100,000$ women in the majority of developed countries (2). However, there have been dramatic reductions in the incidence and mortality of invasive $\mathrm{Ca}$ due to the widespread availability of cytological screening (3). Ca is caused by persistent infection with $\geq 1$ oncogenic human papillomaviruses (HPVs) (4), with the prevalence of HPV infection reaching $99.7 \%$ in cancer patients (5). However, HPV is detected in 3-30\% of asymptomatic controls, indicating the importance of co-factors in the development of cancer of the uterine cervix $(6,7)$.

Malignancies arising from the uterine cervical epithelium are preceded by long periods (occasionally decades) of precancerous dysplastic lesions, usually involving progression from mild, reversible changes to severe irreversible ones (4). The dysplastic lesions were conventionally graded as cervical intraepithelial neoplasia I, II and III, and more recently, as low- or high-grade squamous intraepithelial lesions (LG-SIL or HG-SIL, respectively). Various studies have demonstrated 
that the simultaneous use of colposcopy and cytology as a screening modality exhibits a high sensitivity (95.0-99.4\%) (8).

Mammaliantarget of rapamycin(mTOR)isaserine/threonine protein kinase of the phosphatidylinositol-3-kinase/AKT signaling pathway, with a critical role in controlling cancer cellular growth, metabolism and cell cycle progression (9). When activated, the mTOR signaling pathway regulates ribosomal biogenesis and protein synthesis through phosphorylation and inactivation of the repressor of messenger RNA (mRNA) translation eukaryotic initiation factor 4E (eIF4E) and 4E binding protein 1 (4EBP1) (9), which in turn releases its inhibitory effect on eIF4E. The latter functions as the initiating factor in protein translation machinery. Another downstream effector of the mTOR kinase is the phosphorylation of the ribosomal protein S6 kinase and the subsequent phosphorylation of the ribosomal protein S6 to stimulate protein translation and ribosome biogenesis (9).

A strong body of evidence suggests that the mTOR signaling pathway is activated in a number of solid tumors, including head and neck squamous cell carcinoma (10-13). Notably, mTOR signaling activation has been reported in both HPV-negative and HPV-associated head and neck carcinomas, as well as in cervical carcinoma (13) tissues and cell lines. Furthermore, mTOR inhibitors effectively decreased mTOR activity in vivo and caused a remarkable decrease in tumor burden (13). In addition, overexpression and/or activation of the eIF4E/4EBP1 axis has been reported in numerous human tumors, and has been associated with poor prognosis (14-17).

In the present study, it was hypothesized that the critical downstream effectors of mTOR signaling, 4EBP1 and eIF4E, which are important in protein synthesis control, may be involved in the progression of precancerous lesions and cancer of the uterine cervix. Therefore, the present study investigated the expression patterns of both proteins and correlated the findings with patient characteristics and HPV infection status. The present data demonstrate that high-grade dysplasia and Cas are characterized by overexpression of 4EBP1 and eIF4E proteins, which significantly correlates with oncogenic HPV types, and may provide novel targets for experimental therapies for these patients.

\section{Patients and methods}

Patients. Biopsy specimens were obtained from 73 participants at the 'Elena Venizelou' General Maternity Hospital and 'Alexandra' General Hospital (Athens, Greece) between January 2011 and December 2011. The present study was conducted according to the ethical committee guidelines of the University of Athens (Athens, Greece). Participants were healthy women who had never undergone any kind of therapy for cervical pathology. All women provided written informed consent and answered a behavioral questionnaire (Table I). Women were excluded from the study if they refused HPV DNA testing or if they stated that they had never had sexual intercourse. The present prospective study was restricted to those participants who met the protocol criteria, who were stratified into four groups: i) Normal with non-dysplastic, non-cancerous tissue; ii) LG-SIL; iii) HG-SIL; and iv) Ca. All patients underwent Papanicolaou (Pap) test, HPV DNA test with polymerase chain reaction (PCR) and colposcopic evaluation. Cervical liquid-based cytology samples were gathered, and HPV DNA detection and genotyping plus cytopathological examination using the Bethesda system were performed (7). Cervical samples and biopsy material were tested by PCR for DNA from 14 oncogenic HPV types. The primer sequences are identical to those previously described (18). The cycling conditions were as follows: Incubation at $94^{\circ} \mathrm{C}$ for $10 \mathrm{~min}$, followed by 40 cycles of denaturation for $1 \mathrm{~min}$ at $94^{\circ} \mathrm{C}$, annealing for $1 \mathrm{~min}$ at $55^{\circ} \mathrm{C}$ and elongation for $1 \mathrm{~min}$ at $72^{\circ} \mathrm{C}$. The last cycle was followed by a final extension step of $7 \mathrm{~min}$ at $72^{\circ} \mathrm{C}$. Cervical abnormalities were confirmed by cytological abnormalities on Pap smears or histologically. Colposcopy is important in diagnosis, since this method has the best accuracy for the delineation and biopsy of the truly positive area of the most atypical site (3). From each patient, two biopsies were submitted, one for histological assessment and one for storage at $-80^{\circ} \mathrm{C}$ in liquid nitrogen. All primary samples were transferred in a special collector with liquid nitrogen at the Department of Pathology of the University of Athens. All stored samples in liquid nitrogen were used for western blotting. The Institutional Research Board at the University of Athens approved the study.

Western blot analysis. A total of 40 tissue samples were analyzed by western blotting following total protein extraction. Total proteins were extracted using lysis buffer supplemented with phosphatase and protease inhibitors, as previously described (19). Western blot analysis was also performed using standard methods described previously (19). The monoclonal antibodies used in the present study were specific for eIF4E (catalog no. 9742; dilution, 1:1,000) and 4E-BP1 (catalog no. 9644; dilution, 1:1,000) (Cell Signaling Technology, Inc., Danvers, MA, USA) and $\beta$-actin (Sigma-Aldrich, St. Louis, MO, USA). Detection was performed using enhanced chemiluminescence $\left(A m e r s h a m^{\mathrm{TM}}\right.$ ECL $^{\mathrm{TM}}$ Western Blotting Detection reagent; GE Healthcare Life Sciences, Chalfont, UK). The KARPAS 299 cell line, previously shown to overexpress eIF4E and 4EBP1 proteins (19), served as a positive control in all immunoblots.

Immunohistochemistry. Immunohistochemical analysis was performed on 42 specimens, using previously published methods (19). Briefly, formalin-fixed, paraffin-embedded tissue sections (4- $\mu$ m thick) were obtained, and subsequently deparaffinized in xylene and rehydrated in graded series of alcohol solutions. Monoclonal antibodies specific for total eIF4E (catalog no. 9742; dilution, 1:100) and 4EBP1 (catalog no. 9644; dilution, 1:100) (Cell Signaling Technology, Inc.) were used for immunostaining. Heat-induced antigen retrieval was conducted using preheated target retrieval solution (Dako, Glostrup, Denmark). Based on the intensity of immunostaining in tissues, eIF4E and 4EBP1 expression levels were semi-quantitatively graded as 'strongly positive' $(2+)$, 'weakly positive' (1+) or negative (0). A formalin-fixed, paraffine-embedded cell block with KARPAS 299 cells (a gift from Dr Marshall Kadin, Boston University and Roger Williams Medical Center, Providence, RI, USA) exhibiting overexpression of eIF4E and 4EBP1 proteins (17), served as a positive control. 
Table I. Questionnaire-based clinical and behavioral characteristics of patients included in the present study $(n=73)$.

\begin{tabular}{|c|c|}
\hline Risk factor/categories & $\begin{array}{l}\text { Frequency distribution } \\
\text { at study entry, n (\%) }\end{array}$ \\
\hline \multicolumn{2}{|l|}{ Age group, years } \\
\hline $17-25$ & $12(16.4)$ \\
\hline $26-45$ & $41(56.1)$ \\
\hline$\geq 46$ & $20(27.4)$ \\
\hline \multicolumn{2}{|l|}{ Marital status } \\
\hline Married & $37(50.7)$ \\
\hline Single & $36(49.3)$ \\
\hline \multicolumn{2}{|c|}{ Cigarette smoking, packs/day } \\
\hline Never smoked & $39(53.4)$ \\
\hline$<1$ & $16(22.0)$ \\
\hline 1 & $9(12.3)$ \\
\hline$>1$ & $9(12.3)$ \\
\hline \multicolumn{2}{|c|}{ Age at first sexual intercourse, years } \\
\hline$<15$ & $5(6.8)$ \\
\hline $16-20$ & $47(64.3)$ \\
\hline $21-25$ & $18(24.6)$ \\
\hline$>26$ & $3(4.1)$ \\
\hline \multicolumn{2}{|c|}{ Lifetime number of sexual partners } \\
\hline 1 & $19(26.0)$ \\
\hline$>1$ & $54(74.0)$ \\
\hline \multicolumn{2}{|l|}{ Condom use } \\
\hline Yes & $42(57.5)$ \\
\hline No & $31(42.4)$ \\
\hline \multicolumn{2}{|l|}{ Hormonal contraception } \\
\hline Yes (past) & $20(27.4)$ \\
\hline Yes (current) & $1(1.4)$ \\
\hline No & $52(71.2)$ \\
\hline \multicolumn{2}{|c|}{ History of sexually transmitted infections } \\
\hline Yes & $1(1.4)$ \\
\hline No & $72(98.6)$ \\
\hline \multicolumn{2}{|c|}{ Pregnancy with $\geq 1$ delivery at term } \\
\hline Yes & $42(57.5)$ \\
\hline No & $31(42.4)$ \\
\hline
\end{tabular}

PCR analysis of HPV types. Detection and subtyping of HPV was based on a nested PCR method using suitable sets of primers, which were specific for all 14 high-risk HPV (HR-HPV) types, as described previously (18). Genomic DNA was extracted from paraffin sections of all 73 tissue specimens using standard methods (18).

Statistical analysis. Statistical analysis was performed to compare high (2+) vs. low/absent expression (1+/0) of eIF4E and 4EBP1 with the clinicopathological parameters and HPV status of the patients using the $\chi^{2}$ and Fisher's exact tests. $\mathrm{P}<0.05$ was considered to indicate a statistically significant difference. Statistical calculations were performed using StatView (version 5.0; SAS Institute Inc., Cary, NC, USA).

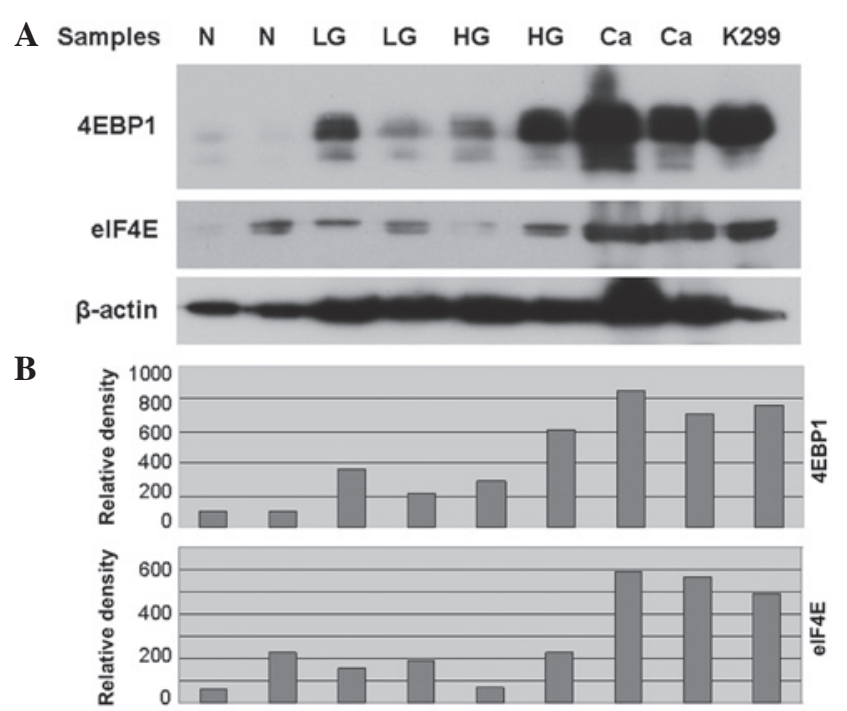

Figure 1. (A) Representative immunoblot of the protein expression levels of 4EBP1 and eIF4E in whole protein extracts from patient samples with $\mathrm{N}$, LG-SIL, HG-SIL and Ca. The KARPAS 299 cell line served as a positive control for high expression levels of both proteins. (B) Bar graphs obtained by densitometric analysis revealed the differences in the expression levels of 4EBP1 and eIF4E among N, LG-SIL, HG-SIL and Ca samples. The Y-axis represents density units, as measured by AlphaImager ${ }^{\circledR}$ software (Proteinsimple, San Jose, CA, USA). N, normal histology; SIL, squamous intraepithelial lesion; LG, low grade; HG, high grade; Ca, cervical cancer; K299, KARPAS 299; 4EBP1, 4E binding protein 1; eIF4E, eukaryotic initiation factor $4 \mathrm{E}$.

\section{Results}

Colposcopy and histology findings. Colposcopy of the cervix was performed after the application of dilute $5 \%$ acetic acid. The ability of histology to define the true level of disease is dependent on accurately biopsied abnormal-appearing lesions (7). However, certain patients had areas of acetowhite changes and vessel abnormalities in the transformation zone. Besides, other patients with certain vaginal infections had also vessels with changes. In all these cases, a biopsy was necessary to establish the origin of acetowhite or angiogenic change. The colposcopic findings in association with histologic diagnosis are summarized in Table II. In total, 68 cases with available data were statistically analyzed. The colposcopy and biopsy groups were stratified into three categories: High, low and negative; 28 specimens were high (HG-SIL or Ca), 29 low (LG-SIL) and 11 negative. Of the 28 biopsy specimens with HG-SIL or Ca, 24 were evaluated as HG-SIL or cancer and only 4 as LG-SIL on colposcopy. Of the 29 biopsy specimens with LG-SIL, 26 were categorized as LG-SIL and only 3 as negative colposcopically . Overall, the association between the colposcopic findings and histologic diagnosis was very strong $\left(\mathrm{P}<0.0001, \chi^{2}\right.$ test). In summary, these data indicate sufficiency in the diagnostic accuracy of colposcopically-directed cervical biopsies in the present study group.

Detection of eIF4E and 4EBP1 protein levels in dysplastic lesions and cancer of the uterine cervix in immunoblots. Western blot analysis revealed high expression levels of eIF4E and 4EBP1 proteins in all cancer specimens tested 
Table II. Expression of 4EBP1/eIF4E proteins in association with patient characteristics.

\begin{tabular}{|c|c|c|c|c|c|c|c|c|c|c|}
\hline \multirow[b]{2}{*}{ Patient } & \multirow[b]{2}{*}{ Age group ${ }^{a}$} & \multirow[b]{2}{*}{ Colposcopy } & \multirow[b]{2}{*}{ Pap test } & \multirow[b]{2}{*}{ Biopsy } & \multirow[b]{2}{*}{ HR-HPV } & \multirow[b]{2}{*}{ HPV16/18 } & \multicolumn{2}{|c|}{ WB } & \multicolumn{2}{|c|}{ IHC } \\
\hline & & & & & & & 4EBP1 & eIF4E & 4EBP1 & eIF4E \\
\hline 1 & 2 & $\mathrm{Neg}$ & $\mathrm{Neg}$ & $\mathrm{Neg}$ & Yes & No & Low & Low & 0 & $1+$ \\
\hline 2 & 1 & Low & LG-SIL & LG-SIL & No & No & Low & Low & $1+$ & 0 \\
\hline 3 & 2 & Low & Susp & LG-SIL & Yes & Yes & Low & Low & $1+$ & $1+$ \\
\hline 4 & 3 & $\mathrm{Neg}$ & $\mathrm{Neg}$ & Neg & No & No & Low & Low & NP & NP \\
\hline 5 & 2 & High & HG-SIL & HG-SIL & NP & NP & High & High & $1+$ & $1+$ \\
\hline 6 & 2 & Low & LG-SIL & LG-SIL & Yes & No & Low & Low & $1+$ & $1+$ \\
\hline 7 & 2 & Low & LG-SIL & LG-SIL & Yes & Yes & Low & Low & $1+$ & $2+$ \\
\hline 8 & 1 & Low & $\mathrm{Neg}$ & LG-SIL & No & No & Low & Low & $2+$ & $1+$ \\
\hline 9 & 2 & Low & LG-SIL & LG-SIL & Yes & Yes & NP & NP & $1+$ & $1+$ \\
\hline 10 & 2 & Low & LG-SIL & HG-SIL & NP & NP & High & High & $2+$ & $2+$ \\
\hline 11 & 2 & Low & LG-SIL & LG-SIL & Yes & Yes & Low & Low & $1+$ & 0 \\
\hline 12 & 2 & Low & LG-SIL & HG-SIL & NP & NP & High & Low & NP & NP \\
\hline 13 & 1 & Low & LG-SIL & HG-SIL & Yes & Yes & Low & Low & NP & NP \\
\hline 14 & 2 & High & HG-SIL & HG-SIL & Yes & No & High & High & $2+$ & $1+$ \\
\hline 15 & 3 & High & NP & SCC & Yes & Yes & High & High & $2+$ & $2+$ \\
\hline 16 & 3 & Low & LG-SIL & LG-SIL & No & No & Low & Low & $1+$ & $1+$ \\
\hline 17 & 2 & Low & LG-SIL & LG-SIL & No & No & Low & Low & NP & NP \\
\hline 18 & 3 & Low & Susp & LG-SIL & Yes & Yes & NP & NP & $1+$ & 0 \\
\hline 19 & 3 & $\mathrm{Neg}$ & $\mathrm{Neg}$ & Neg & Yes & Yes & Low & Low & 0 & 0 \\
\hline 20 & 3 & High & $\mathrm{Neg}$ & $\mathrm{SCC}$ & Yes & No & High & High & $2+$ & $2+$ \\
\hline 21 & 2 & High & HG-SIL & HG-SIL & Yes & Yes & Low & Low & $2+$ & $1+$ \\
\hline 22 & 3 & High & NP & $\mathrm{SCC}$ & Yes & Yes & High & High & $2+$ & $2+$ \\
\hline 23 & 3 & High & NP & SCC & Yes & Yes & High & High & $2+$ & $2+$ \\
\hline 24 & 2 & High & Susp & SCC & NP & NP & High & High & $2+$ & $2+$ \\
\hline 25 & 2 & High & NP & SCC & NP & NP & NP & NP & $2+$ & $2+$ \\
\hline 26 & 1 & High & LG-SIL & HG-SIL & Yes & No & Low & Low & $2+$ & $2+$ \\
\hline 27 & 3 & High & HG-SIL & SCC & Yes & No & High & High & $2+$ & $2+$ \\
\hline 28 & 2 & High & HG-SIL & SCC & NP & NP & High & High & $2+$ & $2+$ \\
\hline 29 & 2 & High & HG-SIL & $\mathrm{SCC}$ & Yes & Yes & $\mathrm{NP}$ & NP & $2+$ & $2+$ \\
\hline 30 & 2 & High & HG-SIL & HG-SIL & Yes & Yes & Low & Low & $2+$ & $2+$ \\
\hline 31 & 2 & High & HG-SIL & HG-SIL & NP & NP & NP & NP & $2+$ & $2+$ \\
\hline 32 & 2 & High & HG-SIL & HG-SIL & Yes & Yes & NP & NP & $2+$ & $2+$ \\
\hline 33 & 2 & High & HG-SIL & $\mathrm{SCC}$ & Yes & Yes & High & High & $2+$ & $2+$ \\
\hline 34 & 2 & Low & HG-SIL & LG-SIL & No & No & High & Low & $1+$ & $1+$ \\
\hline 35 & 3 & High & NP & SCC & Yes & Yes & High & High & $2+$ & $2+$ \\
\hline 36 & 3 & High & NP & $\mathrm{SCC}$ & Yes & Yes & High & High & $2+$ & $2+$ \\
\hline 37 & 2 & Low & HG-SIL & LG-SIL & No & No & Low & Low & $1+$ & $1+$ \\
\hline 38 & 2 & Low & $\mathrm{Neg}$ & Neg & No & No & Low & Low & $1+$ & 0 \\
\hline 39 & 3 & Low & $\mathrm{Neg}$ & Neg & No & No & Low & Low & 0 & $1+$ \\
\hline 40 & 2 & Low & $\mathrm{Neg}$ & Neg & Yes & No & Low & Low & 0 & 0 \\
\hline 41 & 2 & Low & $\mathrm{Neg}$ & Neg & Yes & No & Low & Low & 0 & 0 \\
\hline 42 & 1 & Low & $\mathrm{Neg}$ & Neg & Yes & Yes & Low & Low & $1+$ & 0 \\
\hline 43 & 2 & Low & $\mathrm{Neg}$ & Neg & No & No & Low & Low & $1+$ & $1+$ \\
\hline 44 & 2 & Low & Neg & Neg & Yes & Yes & Low & Low & $1+$ & $1+$ \\
\hline 45 & 3 & High & Neg & HG-SIL & Yes & No & NP & NP & $2+$ & $2+$ \\
\hline 46 & 2 & High & Susp & HG-SIL & Yes & Yes & $\mathrm{NP}$ & NP & $2+$ & $2+$ \\
\hline 47 & 1 & High & LG-SIL & HG-SIL & Yes & Yes & Low & Low & NP & NP \\
\hline 48 & 1 & High & LG-SIL & HG-SIL & Yes & Yes & Low & Low & NP & NP \\
\hline
\end{tabular}

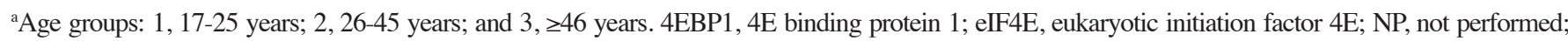
LG, low grade; HG, high grade; SIL, squamous intraepithelial lesion; SCC, squamous cell carcinoma; WB, western blot; IHC, immunohistochemistry; HPV, human papilloma virus; HR, high risk; Neg, negative; Pap, Papanicolaou; Susp, suspicious for human papilloma virus infection or squamous intraepithelial lesion, including atypical squamous cells of undetermined significance, high-grade atypical squamous cells, dyskeratotic cells and hyperkeratosis. 
A
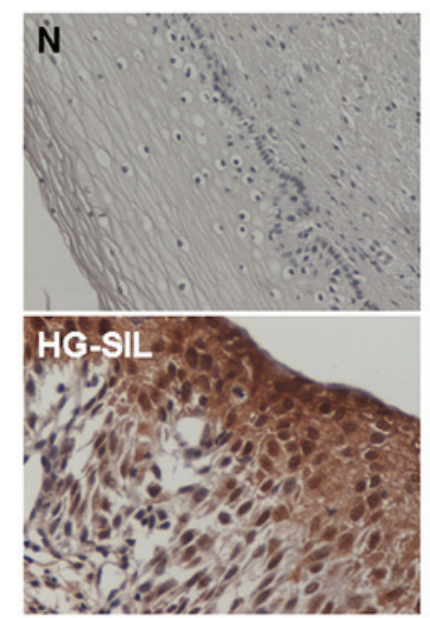

B

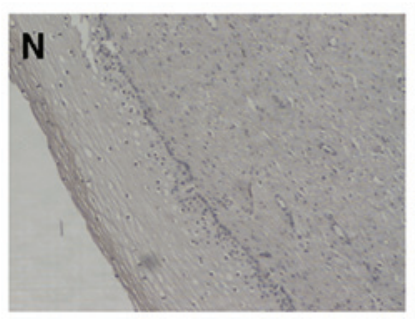

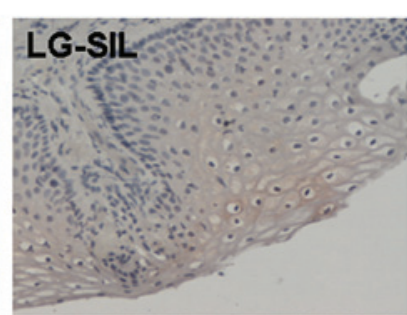
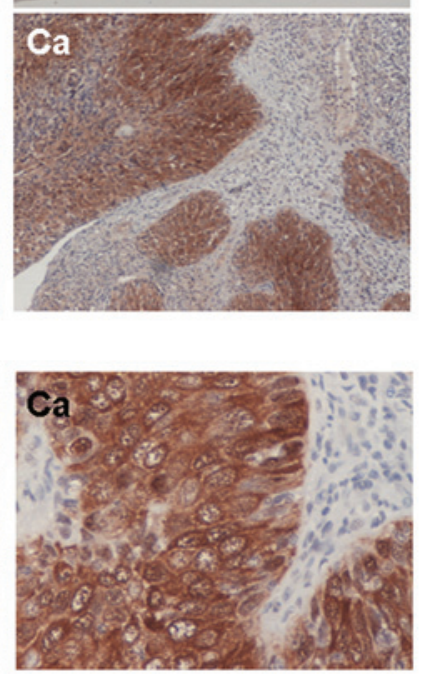

Figure 2. (A) Immunohistochemical expression of 4EBP1 in cervical tissue specimens from patients with N, LG-SIL, HG-SIL and Ca. 4EBP1 expression was minimal in the surrounding stromal tissue, compared with the strong expression observed in specimens with HG-SIL and cancer of the uterine cervix. Original magnification, x100 (N and LG-SIL), x200 (Ca) and x400 (HG-SIL). (B) Representative examples of expression of P-4EBP1. Left, P-4EBP1-negative specimen of cervical epithelium with normal histology. Right, P-4EBP1-positive specimen of carcinoma of the uterine cervix Original magnification, x100 (N) and x400 (Ca). N, normal squamous epithelium; SIL, squamous intraepithelial lesion; LG, low grade; HG, high grade; $\mathrm{Ca}$, cervical cancer; 4EBP1, 4E binding protein 1; P-4EBP1, phosphorylated $4 \mathrm{E}$ binding protein 1 .

(Fig. 1). The eIF4E and 4EBP1 expression level and its association with clinicopathological parameters are summarized in Table II. Among 19 evaluable samples with high-grade dysplasia or carcinomas, overexpression of 4EBP1 and eIF4E proteins was observed in 14 of $19(73 \%)$ and 13 of 19 (68\%) samples, respectively, compared with 1 of $10(10 \%)$ and 0 of $10(0 \%)$ samples in patients with low-grade lesions $(\mathrm{P}=0.0004$ and $\mathrm{P}=0.0017$ for $4 \mathrm{EBP} 1$ and eIF4E, respectively, Fisher's exact test). In addition, strong co-expression of 4EBP1 and eIF4E proteins was also noted in the entire patient group $\left(\mathrm{P}<0.0001, \chi^{2}\right.$ test $)$.

Immunohistochemical expression of eIF4E and 4EBPI in cervical precancerous lesions and cancer. To further elucidate the expression patterns and distribution of eIF4E and 4EBP1 in cervical lesions, immunohistochemical analysis was performed using specific anti-eIF4E and anti-4EBP1 antibodies. This study subgroup included 42 biopsy specimens from patients with normal histology, precancerous lesions (LG-SIL and HG-SIL) and Ca. Histologic slides from a medullary thyroid carcinoma known to overexpress eIF4E and 4EBP1 proteins (20) were used as positive controls. Overexpression (2+) of eIF4E and 4EBP1 was observed in all cancer

A
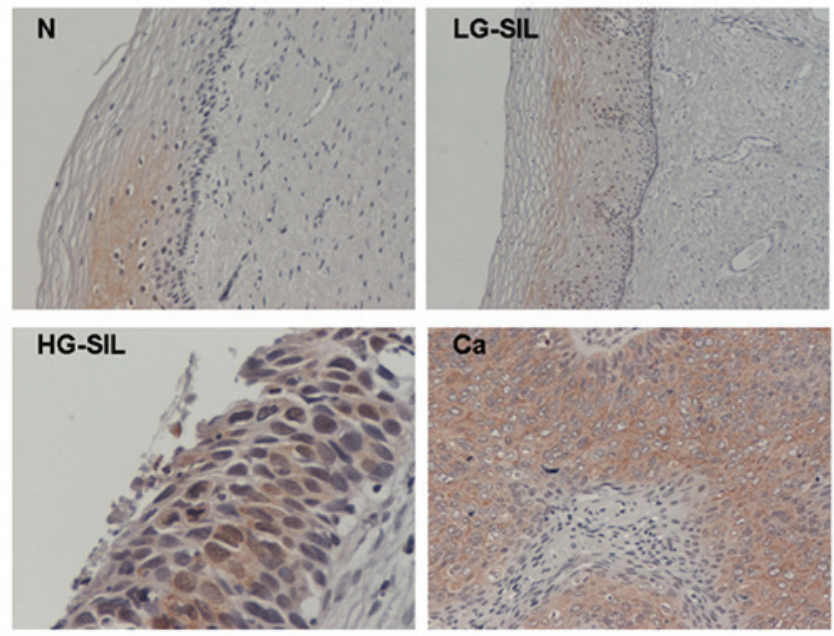

B
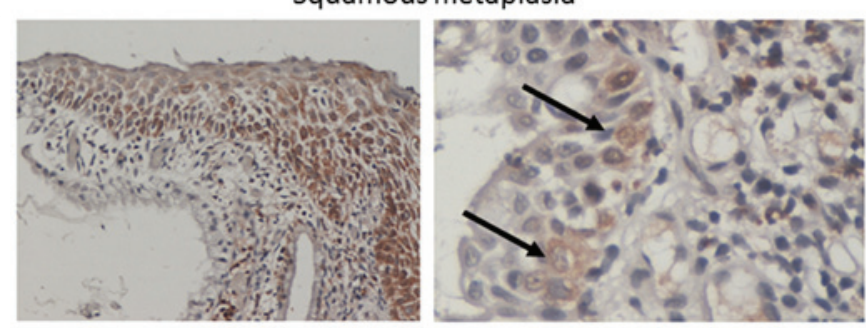

Figure 3. (A) Immunohistochemical expression of eIF4E in cervical tissue specimens from patients with N, LG-SIL, HG-SIL and Ca, being at the highest level in high-grade dysplasia and carcinoma tissue. eIF4E expression was minimal or undetectable in the surrounding stromal tissue. Original magnification, x100 (N and LG-SIL), x200 (Ca) and x400 (HG-SIL). (B) Immunohistochemical expression of eIF4E in cervical tissue specimens with squamous metaplasia and presence of high-risk human papilloma virus type 16. eIF4E was detected at a high level in the majority (left panel) or a subset (right panel) of metaplastic cells in the basal layer. Arrows indicate metaplastic squamous epithelial cells strongly-positive for eIF4E. Original magnification, x100 (left) and x400 (right). N, normal squamous epithelium; SIL, squamous intraepithelial lesion; LG, low grade; HG, high grade; $\mathrm{Ca}$, cervical cancer; eIF4E, eukaryotic initiation factor 4E.

specimens with faint expression in the surrounding stromal area (Fig. 2). By immunohistochemistry, overexpression of 4EBP1 and eIF4E was observed in 20 of 21 (95\%) and 17 of 21 (81\%) samples, respectively, in patients with high-grade dysplasia and carcinomas, compared with 1 of 20 (5\%) and 2 of 20 $(10 \%)$ specimens in patients with low-grade lesions or normal histology ( $\mathrm{P}<0.0001$, Fisher's exact test) (Fig. 2A). In a selected group of 10 4EBP1-positive cases with high-grade dysplasia or carcinomas and 8 4EBP1-negative cases with low-grade lesions or normal histology, immunohistochemistry for the phosphorylated form of 4EBP1 (P-4EBP1) was performed. All (10/10) 4EBP1-positive cases were also positive for P-4EBP1, compared with none $(0 / 8)$ of $4 \mathrm{EBP} 1$-negative cases with low-grade lesions or normal histology $(\mathrm{P}<0.0010$, Fisher's exact test) (Fig. 2B). Of note, in both cases with LG-SIL histology and eIF4E overexpression, squamous metaplasia was present in the lesions (Fig. 3). Immunohistochemical co-expression of the proteins reached significance $\left(\mathrm{P}<0.0001, \chi^{2}\right.$ test).

Associations with HPV status. Detection of any of the 14 HR-HPV types as a group (Fig. 4) was significantly associated with colposcopy findings and histologic diagnosis 
Table III. Correlation between expression of eIF4E and 4EBP1 and HR-HPV.

\begin{tabular}{lccc}
\hline Expression & $\begin{array}{c}\text { HR-HPV } \\
\text { positive }\end{array}$ & $\begin{array}{c}\text { HR-HPV } \\
\text { negative }\end{array}$ & P-value \\
\hline 4EBP1 & & & 0.0400 \\
High & 16 & 1 & \\
Low/neg & 12 & 7 & 0.0100 \\
eIF4E & & & \\
High & 15 & 0 & \\
Low/neg & 13 & 8 & \\
\hline
\end{tabular}

$\mathrm{HR}$, high risk; HPV, human papilloma virus; 4EBP1, 4E binding protein 1; eIF4E, eukaryotic initiation factor 4E; neg, negative.

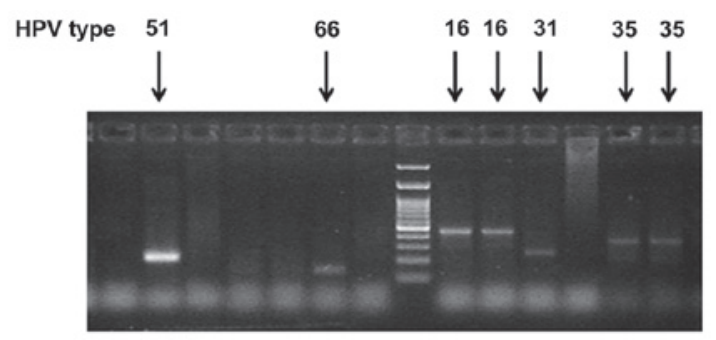

Figure 4. Agarose gel of amplified PCR products of HR-HPV types. Genomic DNA from cervical tissue samples was analyzed by nested PCR for specific genotypes of all $14 \mathrm{HR}-\mathrm{HPV}$ types. Bands of specific size indicate the amplified PCR products for the following HPV types (from left to right): 51 (223bp), 66 (172bp), 16 (457bp), 31 (263bp) and 35 (358bp). HR, high risk; HPV, human papilloma virus; PCR, polymerase chain reaction.

( $\mathrm{P}=0.0040$ and $\mathrm{P}=0.0030$, respectively, Fisher's exact test) in the present study group. Overexpression of 4EBP1 and eIF4E statistically correlated with the presence of HR-HPV oncogenic types $(\mathrm{P}=0.0400$ and $\mathrm{P}=0.0100$, respectively, Fisher's exact test, Table III). In addition, detection of the most oncogenic HPV16/18 types was statistically associated with expression of eIF4E ( $\mathrm{P}=0.0480$, Fisher's exact test) but not with 4EBP1 overexpression ( $\mathrm{P}=0.2000$, Fisher's exact test).

\section{Discussion}

Recent evidence suggests a link between the mTOR signaling pathway and cancer, as the majority of the upstream and downstream components of the mTOR signaling pathway are directly implicated in cancer initiation and progression (9). The present study aimed to investigate whether the expression of the two main downstream effectors of the mTOR signaling pathway, 4EBP1 and eIF4E, may be involved in the progression of dysplasia to cancer of the uterine cervix. Therefore, the expression levels of these proteins and their correlation with the degree of the intraepithelial lesion of the uterine cervix and the presence of HR-HPV types were investigated. The present study demonstrated that 4EBP1 and eIF4E proteins were strongly expressed in all $\mathrm{Ca}$ specimens using western blot analysis and immunohistochemical methods. These findings are in agreement with the results of a previous study demonstrating
mTOR kinase activation in HG-SIL and cancer (21). However, in that study, the expression levels of 4EBP1 and eIF4E proteins were not analyzed. In addition, Faried et al (22) demonstrated that activation (phosphorylation) of AKT and mTOR kinase is detected in $\sim 1 / 2$ of the Cas tested, and represent potential predictive and prognostic indicators in these patients.

The present study also revealed that overexpression of the 4EBP1/eIF4E axis significantly correlates with the degree of cervical dysplasia (SIL). These results are in accordance with the data published by Matthews-Greer et al (23), which reported a progressive increase in immunohistochemical eIF4E expression with increasing degree of cervical dysplastic lesions irrespective of HPV status. In a selected group of cases, the present study also revealed that overexpression of 4EBP1 correlated with its phosphorylation, which is a strong indicator of the activity of the 4EBP1/eIF4E axis and mTOR signaling, which is associated with cancer development and progression (24). More specifically, mTOR complex 1 (mTORC1) positively regulates cell growth, and its inhibition causes cell size decrease at least in part through the 4EBP1/eIF4E axis (24). Inhibition of 4EBP1 due to sequential phosphorylation by mTOR kinase results in the release of eIF4E, which, in its free form, is capable of initiating the translation of various mRNAs, including cell cycle regulators such as cyclin D1 or c-Myc (24). Therefore, eIF4E has been widely recognized to function as an oncogene, whereas 4EBP1 has anti-oncogenic properties (24). For instance, experimental overexpression of eIF4E can cause malignant transformation (24). Upstream of mTOR, several oncogenic mechanisms may operate. Inactivation of tumor suppressors, in particular phosphatase and tensin homolog (PTEN), but also p53 and neurofibromatosis type 1, has been linked to mTOR-regulatory-associated protein of mTOR (raptor) activation (9). Notably, numerous cancer cells without PTEN function and, therefore, with activated AKT signaling, are highly sensitive to the anti-proliferative effects of rapamycin $(25,26)$.

Of note, the only two cases in the present study with LG-SIL and strong expression of eIF4E were associated with squamous metaplasia. It is known that squamous metaplasia may precede epithelial dysplasia of the cervix (7); however, the exact mechanisms underlying this transition are yet unknown. It is tempting to speculate that the mTOR-raptor pathway may be involved in the process, but this merits further investigation.

Besides, it is well established that HPV infection is necessary for cervical carcinogenesis, and therefore, HR-HPV DNA testing is used for screening and secondary prevention $(27,28)$. In the present report, HPV-DNA testing by PCR was used for the detection of HR-HPV types in order to analyze potential associations with the expression of 4EBP1 and eIF4E. Overexpression of 4EBP1 and eIF4E was observed to be significantly associated with the presence of HR-HPV types. This finding suggests that a potential biologic link between the mTOR signaling pathway and oncogenic HPV oncoproteins may exist. This hypothesis is supported by the results of previous studies, which have demonstrated that i) eIF4E is upregulated in cervical neoplasia (23); ii) E6 oncoprotein activates mTORC1 signaling and increases protein synthesis (29); and iii) rapamycin significantly decreases the levels of E7 protein in vitro through the inhibition of mTORC1 (20). Furthermore, a recent study demonstrated that transcription of the $e I F 4 E$ gene is induced by the 
E6 oncoprotein of HPV (30). However, previous studies have reported that activation of AKT may be observed in HPV-negative tumors of the head and neck (31).

Modulation of the mTOR-raptor activity by mTOR inhibitors as well as other targeted therapies has demonstrated promising results in HPV-associated squamous cell carcinomas in vitro (32). Thus, mTOR inhibitors currently tested in clinical trials may indeed have a role in the treatment of carcinomas of the uterine cervix, most likely in combination with chemotherapeutic agents. For instance, use of the single agent temsirolimus has demonstrated activity in cervical carcinoma, with $\sim 2 / 3$ of patients exhibiting stable disease (33). However, despite the increasing knowledge, the role of the mTOR signaling pathway in uterine cervical tumorigenesis requires further investigation. Better understanding of the mTOR pathway may provide the mechanistic basis for novel investigational therapeutic approaches aimed to improve the clinical outcome of women with high-grade dysplasia or cancer of the uterine cervix.

\section{References}

1. Forman D, de Martel C, Lacey CJ, Soerjomataram I, Lortet-Tieulent J, Bruni L, Vignat J, Ferlay J, Bray F, Plummer M, and Franceschi S: Global burden of human papillomavirus and related diseases. Vaccine 30 (Suppl 5): F12-F23, 2012

2. Ferlay J, Shin HR, Bray F, Forman D, Mathers C and Parkin DM: Estimates of worldwide burden of cancer in 2008: GLOBOCAN 2008. Int J Cancer 127: 2893-2917, 2010.

3. World Health Organization. Cervix Cancer Screening. In: IARC Handbook of Cancer Prevention. 1st edition. Vol 10. IARC Press, Lyon, 2005, pp168-173.

4. Muñoz N, Castellsagué X, de González AB and Gissmann L: Chapter 1: HPV in the etiology of human cancer. Vaccine 24 (Suppl 3): S3/1-10, 2006.

5. Walboomers JM, Jacobs MV, Manos MM, Bosch FX, Kummer JA, Shah KV, Snijders PJ, Peto J, Meijer CJ and Muñoz N: Human papillomavirus is a necessary cause of invasive cervical cancer worldwide. J Pathol 189: 12-19, 1999.

6. Burd EM: Human papillomavirus and cervical cancer. Clin Microbiol Rev 16: 1-17, 2003

7. Arends MJ, Buckley CH and Wells M: Aetiology, pathogenesis, and pathology of cervical neoplasia. J Clin Pathol 51:96-103, 1998

8. Richart RM: A modified terminology for cervical intraepithelial neoplasia. Obstet Gynecol 75: 131-133, 1990.

9. Hay N and Sonenberg N: Upstream and downstream of mTOR. Genes Dev 18: 1926-1945, 2004.

10. Surviladze Z, Sterk RT, DeHaro SA and Ozbun MA: Cellular entry of human papillomavirus type 16 involves activation of the phosphatidylinositol 3-kinase/Akt/mTOR pathway and inhibition of autophagy. J Virol 87: 2508-2517, 2013.

11. Gulhati P, Cai Q, Li J, Liu J, Rychahou PG, Qiu S, Lee EY, Silva SR, Bowen KA, Gao T and Evers BM: Targeted inhibition of mammalian target of rapamycin signaling inhibits tumorigenesis of colorectal cancer. Clin Cancer Res 15: 7207-7216, 2009.

12. Clark C, Shah S, Herman-Ferdinandez L, Ekshyyan O, Abreo F, Rong X, McLarty J,Lurie A, Milligan EJ and Nathan CO: Teasing out the best molecular marker in the AKT/mTOR pathway in head and neck squamous cell cancer patients. Laryngoscope 120: $1159-1165,2010$

13. Molinolo AA, Marsh C, El Dinali M, Gangane N, Jennison K, Hewitt S, Patel V, Seiwert TY and Gutkind JS: mTOR as a molecular target in HPV-associated oral and cervical squamous carcinomas. Clin Cancer Res 18: 2558-2568, 2012.

14. Coleman LJ, Peter MB, Teall TJ, Brannan RA, Hanby AM, Honarpisheh H, Shaaban AM, Smith L, Speirs V, Verghese ET, et al: Combined analysis of eIF4E and 4E-binding protein expression predicts breast cancer survival and estimates eIF4E activity. Br J Cancer 100: 1393-1399, 2009.

15. No JH, Jeon YT, Park IA, Kim YB, Kim JW, Park NH, Kang SB, Han JY, Lim JM and Song YS: Activation of mTOR signaling pathway associated with adverse prognostic factors of epithelial ovarian cancer. Gynecol Oncol 121: 8-12, 2011.
16. Inamdar KV, Romaguera JE, Drakos E, Knoblock RJ, Garcia M, Leventaki V, Medeiros LJ and Rassidakis GZ: Expression of eukaryotic initiation factor $4 \mathrm{E}$ predicts clinical outcome in patients with mantle cell lymphoma treated with hyper-CVAD and rituximab, alternating with rituximab, high-dose methotrexate, and cytarabine. Cancer 115: 4727-4736, 2009.

17. Kouvaraki MA, Liakou C, Paraschi A, Dimas K, Patsouris E, Tseleni-Balafouta S, Rassidakis GZ and Moraitis D: Activation of mTOR signaling in medullary and aggressive papillary thyroid carcinomas. Surgery 150: 1258-1265, 2011.

18. Sotlar K, Diemer D, Dethleffs A, Hack Y, Stubner A, Vollmer N, Menton S, Menton M, Dietz K, Wallwiener D, et al: Detection and typing of human papillomavirus by e6 nested multiplex PCR. J Clin Microbiol 42: 3176-3184, 2004.

19. Vega F, Medeiros LJ, Leventaki V, Atwell C, Cho-Vega JH, Tian L, Claret FX and Rassidakis GZ: Activation of mammalian target of rapamycin signaling pathway contributes to tumor cell survival in anaplastic lymphoma kinase-positive anaplastic large cell lymphoma. Cancer Res 66: 6589-6597, 2006.

20. Spangle JM and Münger K: The human papillomavirus type $16 \mathrm{E} 6$ oncoprotein activates mTORC1 signaling and increases protein synthesis. J Virol 84: 9398-9407, 2010.

21. Feng W, Duan X, Liu J, Xiao J and Brown RE: Morphoproteomic evidence of constitutively activated and overexpressed mTOR pathway in cervical squamous carcinoma and high grade squamous intraepithelial lesions. Int J Clin Exp Pathol 2: 249-260, 2009.

22. Faried LS, Faried A, Kanuma T, Sano T, Nakazato T, Tamura T, Kuwano $\mathrm{H}$ and Minegishi T: Predictive and prognostic role of activated mammalian target of rapamycin in cervical cancer treated with cisplatin-based neoadjuvant chemotherapy. Oncol Rep 16: 57-63, 2006.

23. Matthews-Greer J, Caldito G, de Benedetti A, Herrera GA, Dominguez-Malagon $\mathrm{H}$, Chanona-Vilchis $J$ and Turbat-Herrera EA: eIF4E as a marker for cervical neoplasia. Appl Immunohistochem Mol Morphol 13: 367-370, 2005.

24. Zoncu R, Efeyan A and Sabatini DM: mTOR: From growth signal integration to cancer, diabetes and ageing. Nat Rev Mol Cell Biol 12: 21-35, 2011.

25. Neshat MS, Mellinghoff IK, Tran C, Stiles B, Thomas G, Petersen R, Frost P, Gibbons JJ, Wu H and Sawyers CL: Enhanced sensitivity of PTEN-deficient tumors to inhibition of FRAP/mTOR. Proc Natl Acad Sci USA 98: 10314-10319, 2001.

26. Podsypanina K, Lee RT, Politis C, Hennessy I, Crane A, Puc J, Neshat M, Wang H, Yang L, Gibbons J, et al: An inhibitor of mTOR reduces neoplasia and normalizes p70/S6 kinase activity in Pten+/-mice. Proc Natl Acad Sci USA 98: 10320-10325, 2001

27. Ronco G, Biggeri A, Confortini M, Naldoni C, Segnan N, Sideri M, Zappa M, Zorzi M, Calvia M, Accetta G, et al: Health technology assessment report: HPV DNA based primary screening for cervical cancer precursors. Epidemiol Prev 36 (3-4 Suppl 1): e1-e72, 2012.

28. Arbyn M, Ronco G, Anttila A, Meijer CJ, Poljak M, Ogilvie G, Koliopoulos G, Naucler P, Sankaranarayanan R and Peto J: Evidence regarding human papillomavirus testing in secondary prevention of cervical cancer. Vaccine 30 (Suppl 5): F88-F99, 2012

29. Oh KJ, Kalinina A, Park NH and Bagchi S: Deregulation of eIF4E: 4E-BP1 in differentiated human papillomavirus-containing cells leads to high levels of expression of the E7 oncoprotein. J Virol 80: 7079-7088, 2006.

30. Wang S, Pang T, Gao M, Kang H, Ding W, Sun X, Zhao Y, Zhu W, Tang X, Yao Y and Hu X: HPV E6 induces eIF4E transcription to promote the proliferation and migration of cervical cancer. FEBS Lett 587: 690-697, 2013.

31. Won HS, Jung CK, Chun SH, Kang JH, Kim YS, Sun DI and Kim MS: Difference in expression of EGFR, pAkt, and PTEN between oropharyngeal and oral cavity squamous cell carcinoma. Oral Oncol 48: 985-990, 2012.

32. Aderhold C, Faber A, Umbreit C, Birk R, Weiss C, Sommer JU, Hörmann K and Schultz JD: Targeting mTOR and AREG with everolimus, sunitinib and sorafenib in HPV-positive and-negative SCC. Anticancer Res 35: 1951-1959, 2015.

33. Tinker AV, Ellard S, Welch S, Moens F, Allo G, Tsao MS, Squire J, Tu D, Eisenhauer EA and MacKay H: Phase II study of temsirolimus (CCI-779) in women with recurrent, unresectable, locally advanced or metastatic carcinoma of the cervix. A trial of the NCIC Clinical Trials Group (NCIC CTG IND 199). Gynecol Oncol 130: 269-274, 2013. 